Generalized Alvarez lens for correction of laser aberrations

Kai N. LaFortune

December 6, 2004 
This document was prepared as an account of work sponsored by an agency of the United States Government. Neither the United States Government nor the University of California nor any of their employees, makes any warranty, express or implied, or assumes any legal liability or responsibility for the accuracy, completeness, or usefulness of any information, apparatus, product, or process disclosed, or represents that its use would not infringe privately owned rights. Reference herein to any specific commercial product, process, or service by trade name, trademark, manufacturer, or otherwise, does not necessarily constitute or imply its endorsement, recommendation, or favoring by the United States Government or the University of California. The views and opinions of authors expressed herein do not necessarily state or reflect those of the United States Government or the University of California, and shall not be used for advertising or product endorsement purposes.

This work was performed under the auspices of the U.S. Department of Energy by University of California, Lawrence Livermore National Laboratory under Contract W-7405-Eng-48. 


\title{
Generalized Alvarez lens for correction of laser aberrations
}

\author{
K. N. LaFortune
}

December 6, 2004

\section{Introduction:}

The Alvarez lens (US Patent No. 3,305,294 [1]) is a compact aberration corrector. The original design emphasized in the patent consists of a pair of adjacent optical elements that provide a variable focus. A lens system with a variable effective focal length is nothing new. Such systems are widely used in cameras, for example. It is the compactness and simplicity of operation that is the key advantage of the Alvarez lens. All of the complexity is folded into the design and fabrication of the optical elements. As mentioned in the Alvarez patent [1] and elaborated upon in Palusinski et al. [2], if one is willing to fold even more complexity into the optical elements, it is possible to correct higher-order aberrations as well. There is no theoretical limit to the number or degree of wavefront distortions that can be corrected. The only limitation is that there must be a fixed relative magnitude of the aberrations. Independent correction of each component of the higher-order aberrations can not be performed without additional elements and degrees of freedom[3]. Under some circumstances, coupling may be observed between different aberrations. This can be mitigated with the appropriate choice of design parameters. New methods are available today that increase the practicality of making higher-order aberration correctors $[4,5,6]$.

\section{Theory:}
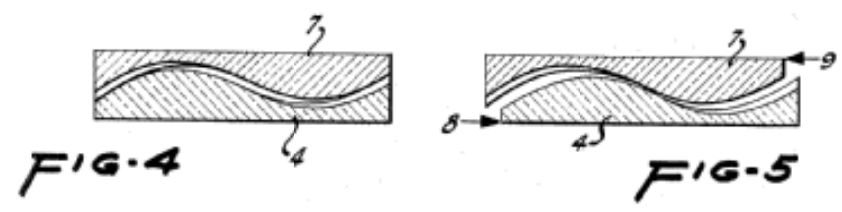

Figure 1: Cross-section illustrations of the Alvarez lens with no offset (left) and lateral displacement (right). The surface figure required for a focus-correcting system is a cubic. This is due to the derivative-like behavior of the system. Correction of any aberration can be achieved by employing a surface whose figure is the integral of the desired correction. Figures reproduced from the Alvarez lens patent[1]

The theory of operation of an Alvarez lens is simple. The lens system consists of two optical elements (see Figure 1). Each with one nominally flat surface and with complimentary figured surfaces with figure $\mathrm{W}(\mathrm{x}, \mathrm{y})$. The two elements are placed nearly in contact with the complimentary surfaces facing each other. In this orientation, the thickness of each element at position $x, y$ is

$$
T+W(x, y) \text { and } T-W(x, y)
$$

where $\mathrm{T}$ is the nominal thickness of the plates. When the two elements are in contact, the system has the optical properties of a plane-parallel plate. The optical path length of a ray passing through the system at a position $x, y$ is simply:

$$
O P L(x, y)=(T+W(x, y)+T-W(x, y))(n-1)=2 T(n-1)
$$

which is independent of the position $x, y$. The variable $n$ is the refractive index of the material used to fabricate the elements.

Even if the two elements are separated slightly, with a gap in between, the optical properties remain essentially the same. With a gap, lateral translation is possible. When the two elements have been translated laterally relative to each other, the system no longer acts like a plane-parallel plate. The optical path length is now

$$
O P L(x, y)=(T+W(x, y)+T-W(x+\Delta x, y+\Delta y))(n-1)
$$




$$
O P L(x, y)=(2 T+W(x, y)-W(x+\Delta x, y+\Delta y))(n-1)
$$

where $\Delta x$ and $\Delta y$ are the translation distances in the $x$ and $y$ directions respectively. The last two terms of Equation 4, if divided by the translation distance, look like the definition of the derivative:

$$
\frac{d f}{d x}=\lim _{\Delta x \rightarrow 0} \frac{f(x)-f(x+\Delta x)}{\Delta x} .
$$

Hence, for small offsets, the wavefront distortions introduced by the Alvarez lens (aside from the 2T delay), are proportional to the derivative of the figure $W(x, y)$. The proportionality constants are the offsets $\Delta x$ and $\Delta y$. Much insight into the performance of an Alvarez lens can be gained by being mindful of this analogy.

As noted by Alvarez[1], it is desirable to translate both elements in opposite directions:

$$
O P L(x, y)=(2 T+W(x+\Delta x / 2, y+\Delta y / 2)-W(x-\Delta x / 2, y-\Delta y / 2))(n-1) .
$$

This provides for the proper cancelling of terms and a simpler relationship between the surface figure and the optical distortion that it introduces. For example, if only one of the elements is translated in a conventional Alvarez lens, there is a nonlinear (a function of $\Delta x^{2}$ ) tilt introduced. This tilt will be small and could be neglected for small $\Delta x$ but can easily be avoided by symmetric translation.

Ignoring the total optical delay $2 T$ for now and looking along just one dimension, the optical path length for a conventional Alvarez lens with a surface figure function of

$$
W(x)=A x+B x^{2}+C x^{3}
$$

becomes, after substitution into Equation 6 and some algebra

$$
O P L(x)=\left(\left(3 C x^{2}+2 B x+A\right) \Delta x+C \Delta x^{3}\right)(n-1) .
$$

The first group of terms looks just like the derivative and contains the desired functionality. If only a quadratic dependence is desired, then the coefficients $A$ and $B$ can be chosen to be zero. The last term is an undesirable term. Fortunately, it only contributes to the total optical delay (no $x$ dependence) and is very small if the displacement is small due to its cubic dependence on $\Delta x$. The non-derivative terms only become more prevalent in the design of higher-order correctors as shall be shown below.

Most systems involve correction along two dimensions, not just one. If $W$ is a cubic along each axis, then a translation along $x$ can introduce an $x^{2}$ (x-astigmatism) term and a translation along $y$ can introduce a $y^{2}$ (y-astigmatism) term. Simultaneous translation along both axes (the xy diagonal) yields an $x^{2}+y^{2}=r^{2}$ (focus) term. Alvarez, in his patents, cleverly oriented the cubic function at 45 degrees relative to the axes so that a translation along a single axis introduces a focus.

The total phase delay introduced, both the static $2 T$ and the offset-dependent terms, has the net effect of increasing the overall path length. In the system of interest, an overall path length change has the effect of changing the laser cavity length. The cavity length change is a fraction of a percent and imperceptible. It is within the noise of the measurement of the cavity length. Even if it were larger, it could be compensated for with the appropriate fine-tuning of the quadratic correction being applied.

The above analysis does not take into account possibly ray offsets due to refraction at steeply figured surfaces. In the system of interest, these offsets will be extremely small. They are much smaller than the errors due to that fact that the corrector plate is not in the same plane or at a conjugate plane of the aberrations for which it is attempting to compensate.

\section{Higher-orders}

Equation 6 can be applied to an analysis of a higher order Alvarez lens. If a separable surface function continues to be used, then it can be investigated along one axis at a time. Take, for example, a 7 th order surface polynomial

$$
W(x)=A x+B x^{2}+C x^{3}+D x^{4}+E x^{5}+F x^{6}+G x^{7} .
$$

Using a pair of optical elements with this surface figure, it is possible to create 6 th order wavefront distortions. After substitution into Equation 6 and a lot of algebra, the functional form of the aberrations are as found in Table 3.

One can see how higher-order polynomial terms mix in to lower-order wavefront aberration terms through high powers of $\Delta x$. Therefore, as long as $\Delta x$ is kept small (i.e., $\Delta x / x<<1$ ), the cross-coupling can be mitigated. The higher the order of the polynomial surface, the smaller $\Delta x / x$ will have to be because of the increasingly large multiplier next to the polynomial coefficients. 


\begin{tabular}{ll} 
Polynomial Term & Seidel Aberration \\
\hline $7 G x^{6} \Delta x$ & \\
$6 F x^{5} \Delta x$ & \\
$\left(35 G \Delta x^{3}+5 E \Delta x\right) x^{4}$ & spherical \\
$\left(20 F \Delta x^{3}+4 D \Delta x\right) x^{3}$ & coma \& trefoil \\
$\left(21 G \Delta x^{5}+10 E \Delta x^{3}+3 C \Delta x\right) x^{2}$ & defocus \& astigmatism \\
$\left(6 F \Delta x^{5}+4 D \Delta x^{3}+2 B \Delta x\right) x$ & tilt \\
$\left(G \Delta x^{7}+E \Delta x^{5}+C \Delta x^{3}+A \Delta x\right)$ & piston
\end{tabular}

Table 1: Tabulated values of polynomial coefficients and corresponding optical aberration of the wavefront introduced by laterally displacing two elements with complimentary surface figures as described in Equation 9.

The displacement needed to introduce a certain wavefront distortion is dependent on the chosen magnitude of the polynomial coefficient. The larger the coefficient, the smaller the total displacement needed to achieve the same distortion. In this way, the maximum $\Delta x / x$ can be kept small enough by increasing the amplitude of the surface modulation, $W(x, y)$. The only trade-off will be the ease of fabrication.

It should be noted that with only two elements and a single degree of freedom (along each axis) only a fixed ratio of aberrations can be corrected (per axis). If the ratio changes, additional elements must be added (either more Alvarez-type plates or a deformable mirror) or the changes must be small enough so that the difference doesn't matter.

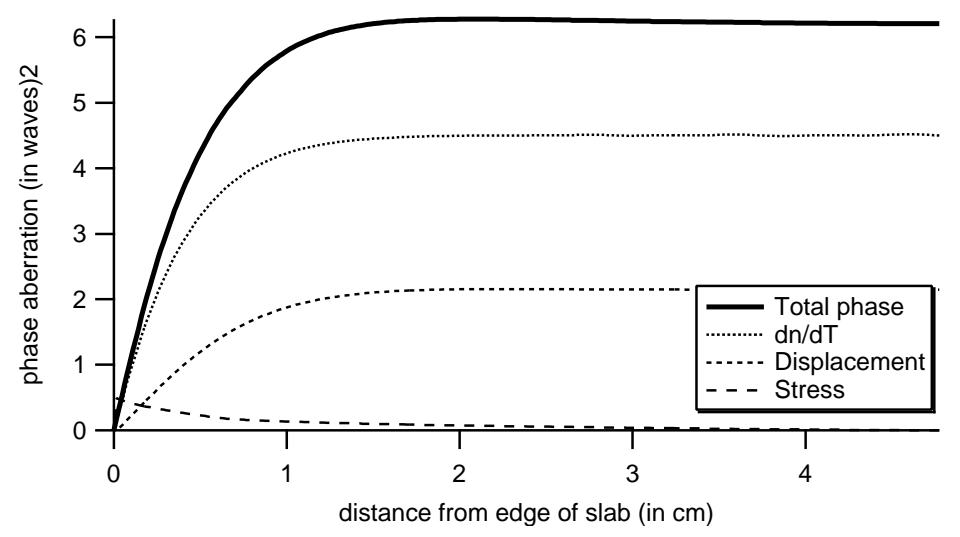

Figure 2: The theoretically estimated phase distortion imparted on the laser wavefront from a single $10 \mathrm{~cm}$ x $10 \mathrm{~cm}$ Nd:GGG slab. The theoretical estimate was benchmarked with experimental data. Due to the symmetry of the distortion, only half of one slice of the curve is shown. The origin is at the edge of the slab, at the interface with the cladding.

\section{SSHCL wavefront}

Before fabrication practicality can be discussed, the desired performance parameters must be addressed. For our particular situation, an Alvarez-type corrector is needed to correct for high-stroke aberrations beyond the range of the existing corrector, a deformable mirror. The nature of the aberrations have been characterized theoretically and experimentally benchmarked. Before fabrication of a corrector could begin, a more rigorous characterization should be performed. The current estimates of the wavefront distortions appear in Figure 2 with a breakdown of the sources of the distortions.

Using the theory from the previous section to design correctors, the residual wavefront error after correcting with an n-th degree polynomial is shown in Figure 3 . The residual wavefront error after correcting with a single monomial term of n-th degree appears in Figure 4. The P-V residuals from the curves in these figures are tabulated in Table 3. Better overall correction is achieved with a polynomial. A monomial correction is more robust because it does not depend upon the ratio of different aberration components remaining the same. The purpose of these figures is to show that with a high-order, low-degree-of-freedom corrector, it is possible to correct for a large fraction of the aberrations in the laser. And with a 6-8 slab laser it is possible to reduce the magnitude of the error to a level that can be corrected with a deformable mirror requiring a stroke of less than 10 microns $\mathrm{P}-\mathrm{V}$. 


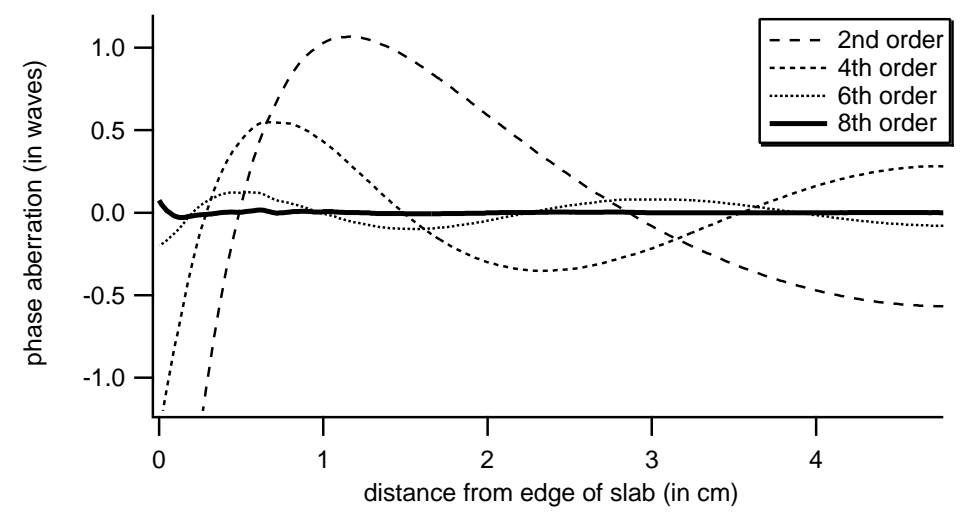

Figure 3: The residual wavefront error from a single slab after correcting with a high-order corrector with the optimum polynomial coefficients. Curves are shown for 2nd, 4th, 6th and 8th degree polynomial corrections. Because of the symmetry of the system, all odd order coefficients are zero.

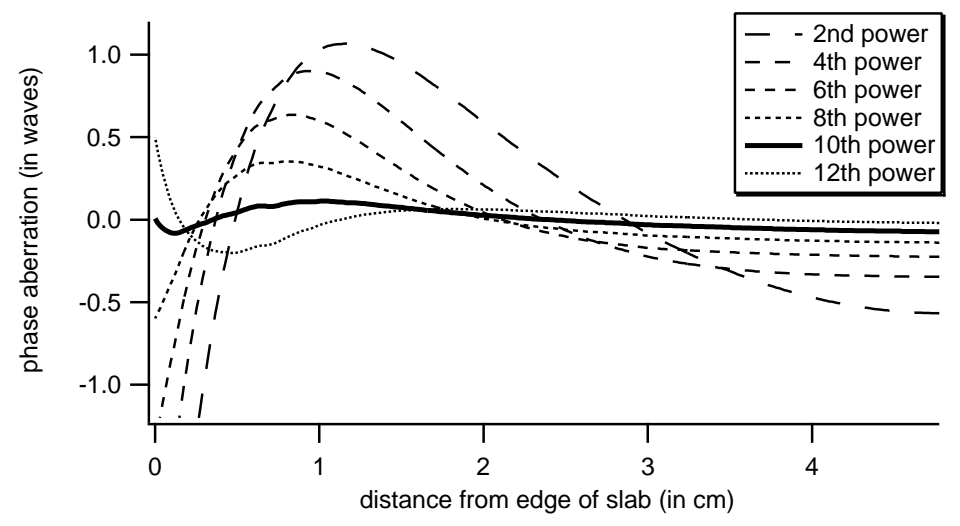

Figure 4: The residual wavefront error from a single slab after correcting with a high-power corrector with the optimum monomial coefficients. Curves are shown for 2nd, 4th, 6th and 8th degree monomial corrections. Because of the symmetry of the system, all odd order coefficients are zero.

The aberrations discussed are the shot-to-shot introduced aberrations. The evolution of the aberrations once the laser has been turned off is more complex. The relative magnitude of the aberrations changes. In particular, the defocus $\left(r^{2}\right)$ term initially grows as heat diffuses towards the edge of the slab before eventually decreasing. The higher-order aberrations immediately decrease. Recall that with only one pair of corrector plates, only a fixed ratio of aberration magnitudes can be corrected. If the laser is to be used in the middle of a cooling phase, these relative changes in the aberrations must be taken into account when designing the optimum correction.

\section{Physical dimensions}

Given the nature of the aberrations as presented in the previous section, the fabrication of the correcting elements can be discussed. Sample surface profiles are illustrated in Figure 5. Since the aberrations are well corrected with a single monomial term of 10th order, an 11th order polynomial is shown. Since the aberrations introduced in the cooling phase of the laser are mostly defocus, and since conventional Alvarez lenses are built as focus correctors, a 3rd order polynomial surface figure is shown. Both tilted $(A \neq 0)$ and non-tilted $(A=0)$ versions are shown to illustrate that a tilted surface figure should probably be used to minimize the amount of surface that needs to be removed to generate the figure. A tilt has no real influence on the wavefront distortions. It will just introduce a piston (overall delay of the wavefront phase), which is unimportant in the laser design. A maximum displacement of the optics of $1 \%$ of the full aperture is allowed. An index of refraction of 1.45 for the substrate material is assumed. This is the index of fused-silica, the most appropriate substrate given its proven performance under high fluences at 1 micron.

The magnitude of the aberrations estimated from preliminary experiments indicate conservatively as much as 6 waves $\mathrm{P}-\mathrm{V}$ of wavefront aberration. That implies for a quadratic correction, which requires about 37 microns P-V of surface figure per wave of distortion, about 2200 microns P-V per slab. In a 6 or 8 slab 


\begin{tabular}{cll} 
Order & $\begin{array}{l}\text { Monomial resid- } \\
\text { ual }\end{array}$ & Polynomial residual \\
\hline 0 & 6.28 waves & 6.28 waves \\
2 & 4.14 waves & 1.63 waves \\
4 & 2.83 waves & 0.90 waves \\
6 & 1.74 waves & 0.22 waves \\
8 & 0.85 waves & 0.03 waves \\
10 & 0.20 waves & $<0.03$ waves \\
12 & 0.48 waves & $<0.03$ waves
\end{tabular}

Table 2: Residual wavefront error for different types of correction to different degrees. Polynomial correction achieves the best overall correction. It demonstrates a best-case scenario in which the nature of the aberrations are fully deterministic. Monomial correction achieves a minimum residual at 10th order due to the particular figure of the aberrations. It more accurately represents a real scenario in which the relative magnitude of different aberrations is not fully known or changes in time like during the cool-down phase of the laser. In such a case, it may be best to only try and correct for a single aberration.

system, that would be between $1.3 \mathrm{~mm}$ and $1.8 \mathrm{~mm}$ of P-V surface figure.

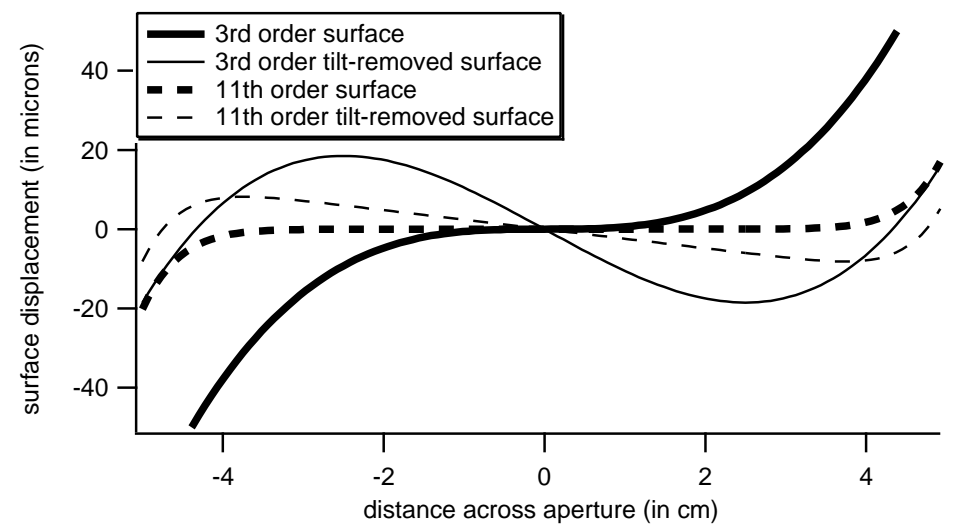

Figure 5: Amount of surface deviation needed per wave of desired correction for two different monomial corrections. A maximum displacement of $\Delta x<x_{\text {total }} / 100$ and a substrate refractive index of 1.45 (fusedsilica) are assumed. The magnitude of the surface deviation for a polynomial correction will be approximately the same.

A potential implementation of an intracavity Alvarez lens for wavefront correction appears in Figure 6 . Placed near one end of the cavity, the Alvarez lens can be designed with any desired nominal correction in its zero-offset position. For example, the illustrated system can be designed with a net focus even if a flat mirror is used. As a result, the combination could be used to replace a concave optic as the end cavity of a laser oscillator. If a deformable mirror is used in place of the flat, increased flexibility can be achieved in correcting wavefront aberrations.

\section{Conclusions}

There are some risks involved in constructing a high-order corrector. First, there is insufficient data as of yet to fully characterize the aberrations that need correcting. Also, there are two modes of aberration growth. There are the shot-to-shot accumulated aberrations. But, there is also a change in the aberrations as the laser cools due to thermal diffusion. The high-order correction applied will have to achieve some compromise in performance between the lasing and cooling states. The fraction of time the slabs will be used in these states must be taken into account to determine the best fixed ratio of aberrations to correct.

There is always some risk when combining multiple control loops. Separation of the control loops for tip-tilt and higher orders has already been demonstrated on the flashlamp-pumped laser system. Off-loading defocus or some other combination of aberrations from the DM would not a paradigm shift for the current AO control system, simply a $50 \%$ increase in the number of simultaneous control loops already running.

The depth of the surface contour that needs to be fabricated is not a trivial task. Polishing starting with 


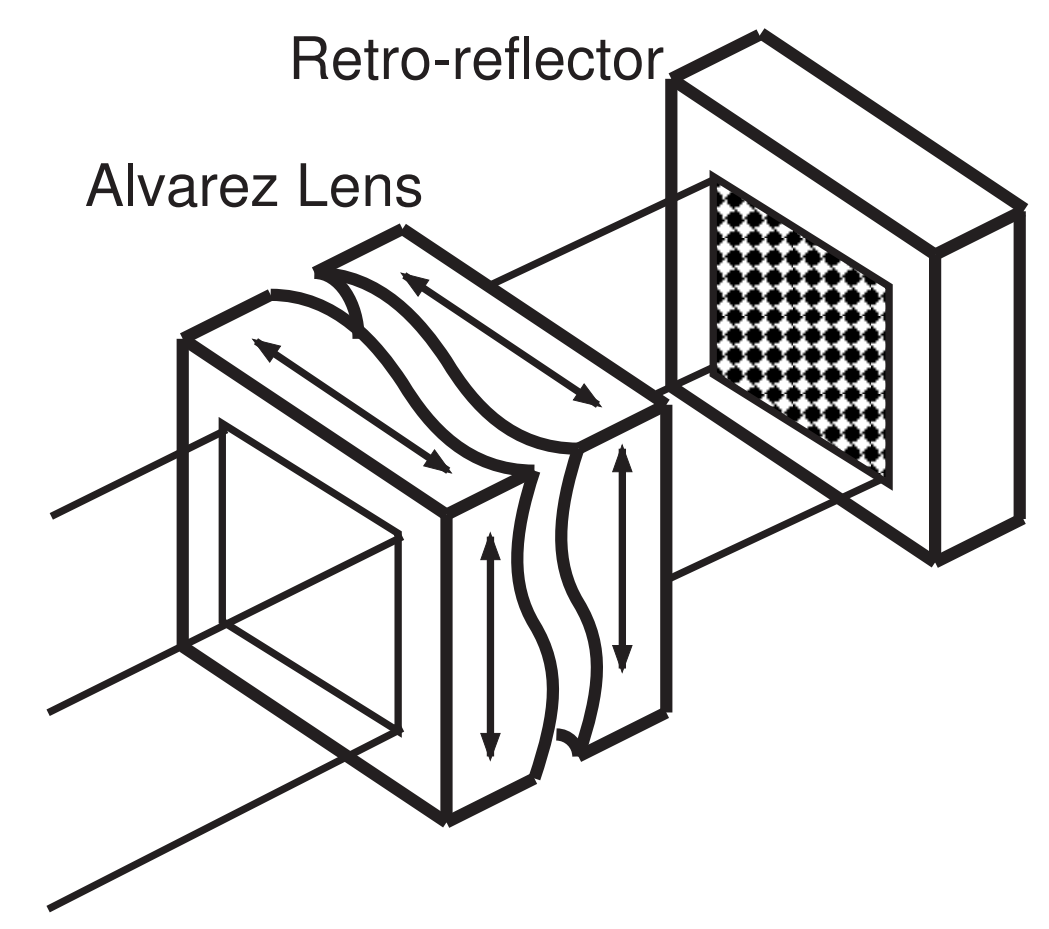

Figure 6: Isometric view of an Alvarez lens in use in combination with a mirror. In such a configuration, the optical path is folded back through the Alvarez lens, thus doubling its influence of the wavefront. The magnitude of its influence is adjusted by translating the two optics in opposing directions along $x$ and $y$ as indicated by the arrows. Thus, correction along each orthogonal direction can be controlled independently.

a flat substrate is probably not practical using the methods suggested above given the time it would take to reach the necessary depth. Either preform molding of the substrates or, a deterministic grinding technique such as diamond turning [7] should probably used to get close to the surface profile before polishing begins. Moreover, interferometrically testing an element with such large surface deviations will be a challenge and will probably involve a combination of surface profilometry and stitching of subaperture interferograms.

\section{Acknowledgements}

Supported by U.S. Army Space and Missile Defense Command (SMDC) This work was performed under the auspices of the U.S. Department of Energy by the University of California, Lawrence Livermore National Laboratory, under Contract no. W-7405-Eng-48. UCRL-TR-208417

\section{References}

[1] L. W. Alvarez, "Two-element variable-power spherical lens," US Patent 3,305,294 (1967).

[2] I. A. Palusinski, J. M. Sasian, and J. E. Greivenkamp, "Lateral Shift Variable Aberration Generators," Appl. Opt. 38, 86-90 (1999).

[3] N. Lopez-Gil, H. C. Howland, B. Howland, N. Charman, and R. Applegate, "Generation of third-order spherical and coma aberrations by use of radially symmetrical fourth-order lenses," J. Opt. Soc. Am. A 15, 2563-71 (1998).

[4] I. M. Barton, S. N. Dixit, L. J. Summers, C. A. Thompson, K. Avicola, and J. Wilhelmsen, "Diffractive Alvarez lens," Opt. Lett. 25, 1-3 (2000).

[5] H. Pollicove, E. Fess, and J. Schoen, "Deterministic manufacturing processes for precision optical surfaces," Proc. SPIE-Int. Soc. Opt. Eng. 5078, 90-6 (2003).

[6] M. Rushford, J. Britten, S. Dixit, C. Hoaglan, M. Aasen, and L. Summers, "Wet-Etch Figuring for Precision Optical Contouring," Appl. Opt. 42, 5706-13 (2003).

[7] M. J. Riedt, "Stretching the optical envelope," Photonics Spectra 35, 130-2 (2001). 